\title{
Epidemiologic Shift in Candidemia Driven by Candida auris, South Africa, 2016-2017 1
}

\author{
Erika van Schalkwyk, ${ }^{2}$ Ruth S. Mpembe, Juno Thomas, Liliwe Shuping, Husna Ismail, \\ Warren Lowman, Alan S. Karstaedt, Vindana Chibabhai, Jeannette Wadula, \\ Theunis Avenant, Angeliki Messina, Chetna N. Govind, Krishnee Moodley, \\ Halima Dawood, Praksha Ramjathan, Nelesh P. Govender, ${ }^{2}$ for GERMS-SA
}

Candida auris is an invasive healthcare-associated fungal pathogen. Cases of candidemia, defined as illness in patients with Candida cultured from blood, were detected through national laboratory-based surveillance in South Africa during 2016-2017. We identified viable isolates by using mass spectrometry and sequencing. Among 6,669 cases (5,876 with species identification) from 269 hospitals, 794 (14\%) were caused by $C$. auris. The incidence risk for all candidemia at 133 hospitals was 83.8 (95\% Cl 81.286.4 ) cases $/ 100,000$ admissions. Prior systemic antifungal drug therapy was associated with a $40 \%$ increased adjusted odds of $C$. auris fungemia compared with bloodstream infection caused by other Candida species (adjusted odds ratio 1.4 [95\% Cl 0.8-2.3]). The crude in-hospital case-fatality ratio did not differ between Candida species and was $45 \%$ for $C$. auris candidemia, compared with $43 \%$ for non-C. auris candidemia. $C$. auris has caused a major epidemiologic shift in candidemia in South Africa.

Nince 2009, when the first case of Candida auris infec$\checkmark$ tion was identified in South Africa, the number of laboratory-confirmed cases has increased exponentially (1).

Author affiliations: National Institute for Communicable Diseases, Johannesburg, South Africa (E. van Schalkwyk, R.S. Mpembe, J. Thomas, L. Shuping, H. Ismail, N.P. Govender); Vermaak \& Partners-Pathcare Pathologists, Johannesburg (W. Lowman); Wits Donald Gordon Medical Centre, Johannesburg (W. Lowman); University of the Witwatersrand, Johannesburg (W. Lowman,

A.S. Karstaedt, V. Chibabhai, J. Wadula, A. Messina,

N.P. Govender); University of Pretoria and Kalafong Provincial Tertiary Hospital, Pretoria, South Africa (T. Avenant); Netcare Hospitals Ltd, Johannesburg (A. Messina); Lancet Laboratories, Durban, South Africa (C.N. Govind, K. Moodley); University of KwaZulu-Natal, Durban (C.N. Govind, H. Dawood, P. Ramjathan); Grey's Hospital, Pietermaritzburg, South Africa (H. Dawood); National Health Laboratory Service, Johannesburg (V. Chibabhai, J. Wadula, P. Ramjathan)

DOI: https://doi.org/10.3201/eid2509.190040
This multidrug-resistant fungal pathogen emerged worldwide, appearing almost simultaneously on 6 continents, causing invasive disease and protracted healthcare-associated outbreaks (2-5). The reported crude case-fatality ratio among patients with invasive $C$. auris infections is high, although the attributable mortality rate has not been determined $(3,6)$. C. auris persists on surfaces, is transmitted among patients in the healthcare environment, forms biofilms, and resists routinely used environmental cleaning agents (7-10). Candida spp. are a common cause of bloodstream infections and were responsible for $13 \%$ (95\% CI 6\%-26\%) of healthcare-associated bloodstream infections according to a 2015 US point-prevalence survey (11). C. parapsilosis was the dominant species causing candidemia according to a national survey in South Africa conducted during 2009-2010 (12). Patients at risk for candidemia in general are the critically ill (especially premature neonates) and those with serious underlying illnesses (e.g., diabetes mellitus and hematologic malignancies), prior or prolonged exposure to broad-spectrum antimicrobial drugs, and invasive medical and surgical interventions (13). Previously described characteristics associated with candidemia among adults in South Africa included abdominal surgery, trauma, diabetes mellitus, cancer, and HIV infection (14). C. auris is thought to occupy a similar niche in the healthcare environment as $C$. parapsilosis because both organisms colonize human skin and adhere to healthcare surfaces and devices. Clinical risk factors for $C$. auris infection would be expected to be similar to those for C. parapsilosis infection, but these factors are largely reported from several small case series. Risk factors for $C$. auris candidemia (compared with other species) among patients admitted to 27 intensive care

${ }^{1}$ Preliminary results from this study were presented at the Federation of Infectious Diseases Societies of Southern Africa (FIDSSA) conference (oral abstract no. 8,382), November 9-11 2017, Cape Town, South Africa.

${ }^{2}$ These authors contributed equally to this article. 
units in India included underlying respiratory disease, vascular surgery, having a urinary catheter in situ, prior antifungal drug exposure, and a low APACHE II score at admission (6). In South Africa, most reported cases of $C$. auris colonization or invasive disease occurred in older patients (median age 60 years) (1) (R.E. Magobo, National Institute for Communicable Diseases [NICD], South Africa, pers. comm., 2019 Jul 1). To inform infection prevention and empiric antifungal treatment strategies, we used national surveillance data for South Africa to estimate the total incidence risk for candidemia and the proportion of candidemia cases caused by $C$. auris and to determine factors associated with $C$. auris candidemia compared with other Candida species,

\section{Materials and Methods}

\section{Surveillance for Candidemia}

From January 1, 2016, through December 31, 2017, we conducted active national laboratory-based surveillance for candidemia by using the NICD GERMS-SA surveillance platform. We requested that Candida species from any episode of bloodstream infection, with an accompanying laboratory report (including basic patient demographic data), be submitted from all clinical microbiology laboratories within the National Health Laboratory Service (NHLS), a national public-sector laboratory network, and from all pathology laboratory practices in the private sector. We have previously described the methods used by private and NHLS laboratories for species identification (1). Isolates were sent to the NICD's Mycology Reference Laboratory for confirmation of identification and antifungal drug susceptibility testing. In addition, surveillance officers (nurses or pharmacists) collected basic clinical and demographic data on standardized electronic case report forms at 22 public-sector and 3 private-sector enhanced surveillance sites, all of which were large acutecare hospitals. We did not collect sufficient data to define severity of illness scores (e.g., APACHE II or McCabe scores). We conducted retrospective audits to ensure complete case ascertainment.

We extracted line list data from the laboratory information systems of NHLS and private laboratories, compared those data with reported cases, deduplicated the data (by using patient name, surname, date of birth, hospital number, and specimen collection date), and added missing cases to the surveillance database. For cases detected by audit, we recorded the Candida species identification reported by the reporting laboratory. In 2013, the estimated number of beds in private-sector hospitals nationwide was 34,572 , of which $45 \%$ were located in Gauteng Province, the most economically active and densely populated province of South Africa (15).

\section{Case Definitions}

We defined a case of candidemia as illness in any patient at a healthcare facility in South Africa who had Candida species isolated from a blood culture specimen processed by an NHLS or private-sector diagnostic laboratory. We defined a confirmed case of $C$. auris candidemia as illness in a patient with an isolate confirmed as $C$. auris at NICD, regardless of the referring laboratory's initial identification. We also included probable cases for which the referring laboratory identified $C$. auris or Candida haemulonii but a viable isolate was not available for confirmation at NICD. Multiple Candida isolates cultured within 30 days of the first positive blood culture specimen were included in a single case. We classified cases of candidemia into 2 groups on the basis of NICD identification (or the referring laboratory's identification if a viable isolate was not available): C. auris and non-C. auris candidemia.

\section{Reference Laboratory Methods}

Isolates were submitted to NICD on Dorset transport medium (Diagnostic Media Products, http://www.nhls.ac.za). For viable isolates, species-level identification was confirmed by using matrix-assisted laser desorption/ionization time-of-flight (MALDI-TOF) mass spectrometry (Bruker Corporation, https://www.bruker.com). We amplified and sequenced the internal transcribed spacer or D1/D2 region of the ribosomal gene for isolates when MALDI-TOF mass spectrometry did not yield a score $\geq 2$.

\section{Statistical Analyses}

We calculated the overall incidence risk for candidemia for hospitals for which admissions data were available, stratified by healthcare sector, by dividing the total number of new cases of candidemia by the total number of hospital admissions (i.e., number of persons at risk) in each sector for the 2-year period. We also calculated healthcare facility incidence risk per hospital when admission denominator data were available. We obtained admissions data by directly approaching private hospital groups and through the GERMS-SA surveillance platform for public-sector hospitals. We used ArcGIS mapping software (https://www.esri. com) to plot the location and number of $C$. auris candidemia cases at hospitals in Gauteng Province and used inverse distance-weighted interpolation to map hotspot hospitals, which we defined as those with $>10$ reported cases of $C$. auris candidemia during the 2-year period.

We hypothesized a priori that systemic azole exposure was associated with candidemia caused by $C$. auris rather than other Candida species. Distinguishing cases of C. auris candidemia from those caused by other species is important to physicians choosing an empiric antifungal treatment regimen for suspected candidemia and to infection prevention and control practitioners for rapid identification 
of cases requiring contact precautions. We used multivariable logistic regression to assess this association among patients admitted to 25 enhanced surveillance sites.

We compared proportions between groups by using a $\chi^{2}$ or Fisher exact test. We compared medians by using a Wilcoxon rank-sum test.

\section{Ethics}

NICD obtained annual approval for GERMS-SA laboratory-based surveillance from the human research ethics committees of several universities in South Africa. Patients from whom surveillance data were collected prospectively through interview provided written informed consent.

\section{Results}

During the 2-year surveillance period, 6,669 cases of candidemia (6,629 first and 40 recurrent episodes) were detected across South Africa at 103 public-sector and 166 privatesector hospitals $(2,529$ cases [38\%] in the public sector, 4,140 cases $[62 \%]$ in the private sector). Of the 6,669 cases, viable isolates were identified to species level at NICD for $3,020(45 \%)$ cases. Species identification was available for a further 2,856 cases $(2,842$ from private laboratories, 14 from NHLS laboratories). Among 5,876 cases with a species-level identification, 794 (14\%) were caused by $C$. auris and 5,082 (86\%) by other Candida species (Figure
1). The most common Candida species in the non-C. auris group were $C$. parapsilosis $(2,600$ [44\%]), C. albicans $(1,353[23 \%]), C$. glabrata $(598$ [10\%]), C. tropicalis $(140$ [2\%]), and C. krusei (98 [2\%]). Twenty-nine cases had a mixed episode of candidemia caused by $C$. auris and another Candida species (mostly C. parapsilosis [21 cases]).

The total incidence risk for candidemia (expressed as cases/100,000 hospital admissions) at 115 private-sector and 18 public-sector hospitals with available admissions data was $71.2(95 \%$ CI 68.6-73.8) in the private sector and 149.5 (95\% CI 141.1-158.1) in the public sector, for a total of 83.8 (95\% CI 81.2-86.4]) (Table 1). Incidence risk for C. auris was $13.6(95 \%$ CI $12.4-14.8)$ in the private sector, compared with 6.9 (95\% CI 5.2-9.0) in the public sector; incidence risk ratio was 1.96 (95\% CI 1.4-2.6). Individual healthcare facility incidence risk ranged from 2.6 to 375 for C. parapsilosis, 1.3 to 221 for C. albicans, 0.9 to 154 for $C$. auris, and 1.7 to 107 for C. glabrata.

We received 4,236 isolates from 70 NHLS laboratories and 4 amalgamated private-sector pathology practices, and we identified an additional 3,307 cases (with 3,373 corresponding isolates) by retrospective audits. Of the 400 confirmed viable $C$. auris isolates received, $258(65 \%)$ had an initial identification of $C$. auris.

Among 435 patients with $C$. auris candidemia for whom data were available (including 9 patients with probable

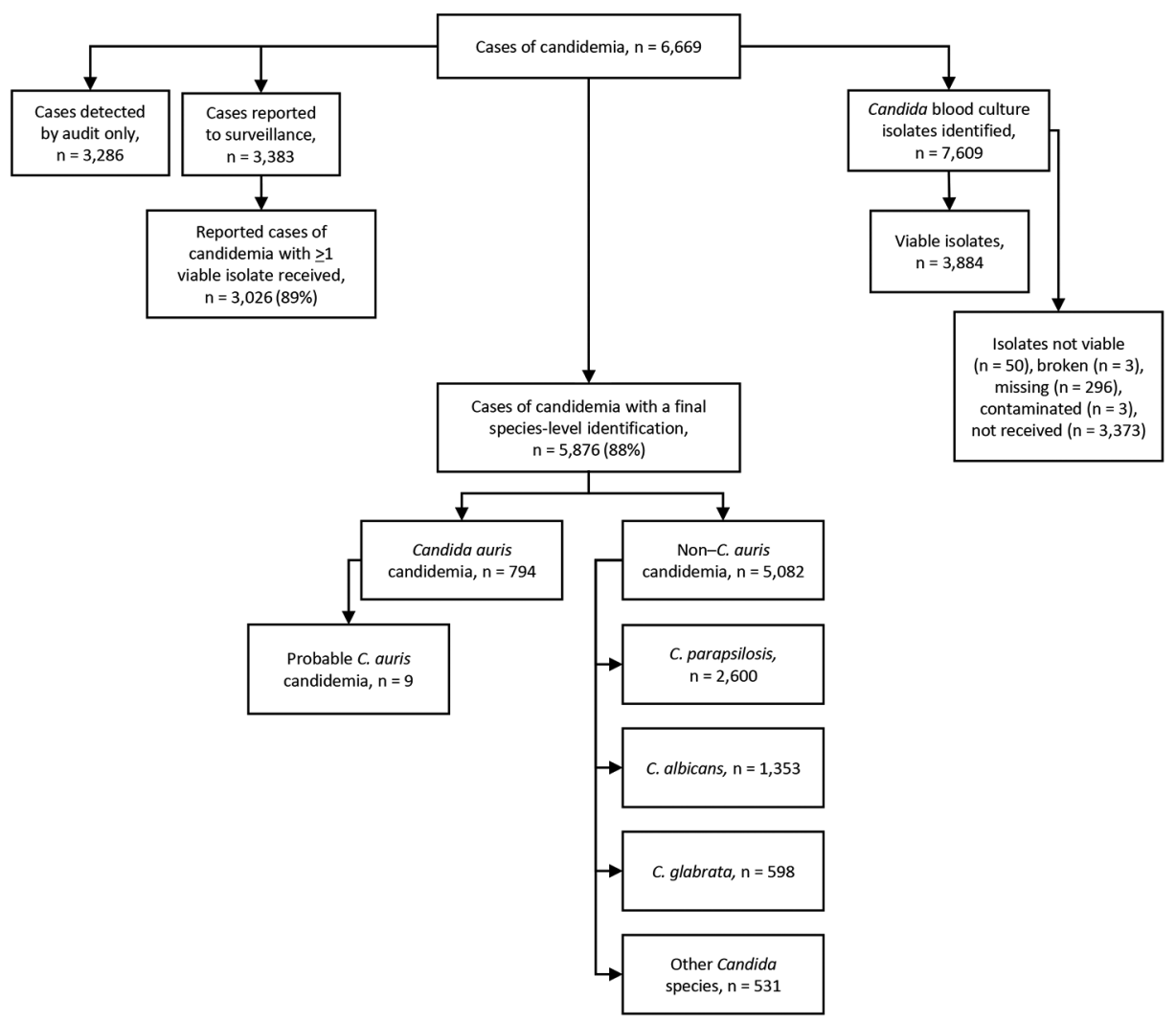

Figure 1. Flowchart showing numbers of candidemia cases detected by national surveillance and Candida species identified, South Africa, 2016-2017. 
Table 1. Incidence risk for candidemia at a limited number of public- and private-sector hospitals with available admissions data, by Candida species and healthcare sector, South Africa, 2016-2017*

\begin{tabular}{|c|c|c|c|c|c|}
\hline Candida species & $\begin{array}{l}\text { No. cases } \\
\text { at } 133 \\
\text { hospitals }\end{array}$ & $\begin{array}{c}\text { Total incidence risk } \dagger \\
(95 \% \mathrm{Cl})\end{array}$ & $\begin{array}{c}\text { Incidence risk at } 18 \\
\text { public-sector hospitals } † \\
(95 \% \mathrm{Cl})\end{array}$ & $\begin{array}{c}\text { Incidence risk at } 115 \\
\text { private-sector hospitals } \\
(95 \% \mathrm{Cl})\end{array}$ & $\begin{array}{l}\text { Incidence risk ratio, } \\
\text { private sector:public } \\
\text { sector }(95 \% \mathrm{Cl})\end{array}$ \\
\hline C. parapsilosis & 1,657 & $32.98(31.3-34.6)$ & $27.98(24.4-31.9)$ & $33.94(32.2-35.8)$ & $1.21(1.0-1.4)$ \\
\hline C. albicans & 735 & $14.63(13.5-15.8)$ & $34.55(30.6-38.9)$ & $10.82(98.5-11.9)$ & $0.31(0.2-0.4)$ \\
\hline C. auris & 628 & $12.50(11.5-13.6)$ & $6.93(5.2-9.0)$ & 13.57 (12.4-14.8) & $1.96(1.4-2.6)$ \\
\hline C. glabrata & 352 & $7.01(6.2-7.8)$ & $12.13(9.8-14.8)$ & $6.02(5.31-6.9)$ & $0.50(0.3-0.7)$ \\
\hline Other & 308 & $6.13(5.4-6.9)$ & $13.25(10.8-16.1)$ & $4.77(4.1-5.5)$ & $0.36(0.2-0.5)$ \\
\hline Totalł & 4,209 & $83.78(81.2-86.4)$ & $149.46(141.1-158.1)$ & $71.20(68.6-73.8)$ & $0.48(0.4-0.6)$ \\
\hline $\begin{array}{l}\text { total of } 529 \mathrm{ca} \\
\text { mbers are inclu }\end{array}$ & e total $r$ & . & & 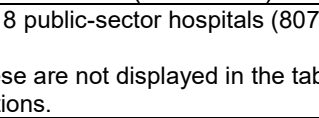 & \\
\hline
\end{tabular}

C. auris infection), the median age was 54 years (interquartile range [IQR] 34-67 years), compared with a median of 27 years (IQR 0-57 years) among 4,050 patients with non-C. auris candidemia $(\mathrm{p}<0.001)$ (Table 2; Figure 2). Neonates comprised the largest proportion of patients with non- $C$. auris candidemia $(1,015 / 4,050 ; 25 \%)$, whereas only 20 cases $(5 \%)$ in the $C$. auris group were in neonates (Table 2). Of patients with C. auris, $61 \%$ (284/463) were male; $54 \%$ $(1,729 / 3,216)$ were male in the non-C. auris group.

Most $(86 \%, 680 / 794)$ cases of $C$. auris candidemia were from hospitals in Gauteng Province and $88 \%(695 / 794)$ from private-sector facilities, compared with $60 \%(3,549 / 5,875)$ in Gauteng Province and 59\% $(3,445 / 5,875)$ from private-sector facilities among non$C$. auris cases. Cases of $C$. auris candidemia were diagnosed at 14 public-sector and 67 private-sector hospitals (Figure 3), most of which are located in Gauteng Province (Figure 4). Among these, 25 hospitals had $>10$ cases of $C$. auris candidemia during the 2-year period (meeting our definition of hotspot hospitals); the largest absolute number of cases was reported from a large private hospital and another large academic teaching hospital. However, incidence risk for $C$. auris candidemia was highest in a smaller private-sector hospital (13 cases/8,431 admissions [154 cases/100,000 admissions]). Of the 20 hospitals with the highest incidence, 19 were private-sector facilities. Several small outbreaks occurred at these hotspot hospitals, but in different wards within each hospital (data not shown).

We collected clinical data for 2,067 patients at enhanced surveillance sites, including 535 patients whose isolates were not identified at the species level. Most patients with $C$. auris bloodstream infections had received prior ( $\leq 14$ days before diagnosis) systemic antimicrobial drug therapy $(77 / 94[82 \%])$, and 30/95 (32\%) had received prior systemic antifungal drug therapy. Of the 30 patients with prior antifungal therapy, 16 had received azoles, 7 had received amphotericin $\mathrm{B}$, and 13 had received echinocandins. Among 105 patients with $C$. auris candidemia for whom clinical data were available, the median length of hospitalization before onset of candidemia was 28 days (IQR 15-46 days), compared with 12 days (IQR 5-23 days) among 1,852 patients with non-C. auris candidemia $(p<0.001)$. Approximately one third $(32 / 105[31 \%])$ of patients with $C$. auris candidemia spent $>6$ weeks in hospital before the first positive blood culture was obtained. Seventy-seven (74\%) patients with $C$. auris infection had been hospitalized in the past year, and $110(88 \%)$ patients were admitted to an intensive care unit at some point during their current hospital stay. Eleven (26\%) of 43 patients with $C$. auris candidemia were HIV-seropositive, similar to patients infected with other Candida species (251/972; $26 \%$ ). The crude in-hospital case-fatality ratio did not differ between Candida species and was $45 \%$ for $C$. auris candidemia, compared with $43 \%$ for non-C. auris candidemia $(\mathrm{p}=0.6)($ C. albicans, $50 \%$; C. parapsilosis, 32\%; C. glabrata, 51\%) (Table 2).

Prior systemic antifungal drug therapy was associated with $40 \%$ increased adjusted odds of $C$. auris fungemia; nevertheless, an effect ranging from a $20 \%$ decrease to a 2.5 -fold increase is also consistent with our data (adjusted odds ratio [aOR] 1.4 [95\% CI 0.8-2.3]). A central venous catheter in situ also independently increased the odds of C. auris infection 2-fold (aOR 1.8 [95\% CI 1.05-3.01]). Admission to a private-sector facility increased the odds of $C$. auris candidemia 3-fold (aOR 2.7 [95\% CI 1.5-4.7]). Older patients (aOR 1.01 [95\% CI 1.01-1.03] for every year) with longer hospitalization before the first positive blood culture (aOR 1.01 [95\% CI 1.01-1.02] for every day admitted) were more likely to have $C$. auris fungemia.

To understand whether inherent differences between healthcare sectors influenced risk factors, we stratified C. auris data by healthcare sector (Table 3). In the public sector, prior antifungal drug therapy (especially azole therapy) was associated with 2-fold increased odds of $C$. auris bloodstream infection (aOR 2.0 [95\% CI 1.0-3.9]; $\mathrm{p}=0.04)$ after adjustment for patient age, sex, length of hospital stay, previous hospitalization, and presence of a central venous catheter in situ. 
Table 2. Demographic and clinical characteristics of 6,669 patients with candidemia caused by Candida auris compared with other Candida species, South Africa, 2016-2017*

\begin{tabular}{|c|c|c|c|c|c|c|}
\hline Characteristics & All candidemia & C. auris & Non-C. auris & C. parapsilosis & C. albicans & C. glabrata \\
\hline No. case-patients & 6,669 & 794 & 5,875 & 2,600 & 1,353 & 598 \\
\hline $\begin{array}{l}\text { Systemic antifungal drug therapy } \\
<14 \text { d before positive culture }\end{array}$ & $317 / 1,829(17.3)$ & $30 / 95(31.6)$ & $287 / 1,734(16.6)$ & $108 / 477(22.6)$ & $36 / 441(8.2)$ & $11 / 166(6.6)$ \\
\hline $\begin{array}{l}\text { Azole } \\
\text { Polyene/amphotericin B } \\
\text { Echinocandin }\end{array}$ & $\begin{array}{c}219 / 317(69.1) \\
38 / 317(12) \\
79 / 317(24.9) \\
32(0-58)\end{array}$ & $\begin{array}{c}16 / 30(53.3) \\
7 / 30(23.3) \\
13 / 30(43.3) \\
54(34-67)\end{array}$ & $\begin{array}{c}203 / 287(70.7) \\
31 / 287(10.8) \\
66 / 287(23) \\
27(0-57)\end{array}$ & $\begin{array}{c}72 / 108(66.7) \\
12 / 108(11.1) \\
27 / 108(25) \\
24(0-58)\end{array}$ & $\begin{array}{c}30 / 36(83.3) \\
5 / 36(13.9) \\
2 / 36(5.6) \\
24(0-56)\end{array}$ & $\begin{array}{c}9 / 11(81.8) \\
0 / 11(0) \\
2 / 11(18.2) \\
54(32-67)\end{array}$ \\
\hline Sex & & & & & & \\
\hline $\begin{array}{l}\text { Men and boys } \\
\text { Women and girls }\end{array}$ & $\begin{array}{c}2,013 / 3,679 \\
(54.7) \\
1,666 / 3,679 \\
(45.3) \\
\end{array}$ & $\begin{array}{c}284 / 463 \\
(61.3) \\
179 / 463 \\
(38.7) \\
\end{array}$ & $\begin{array}{c}1,729 / 3,216 \\
(53.8) \\
1,487 / 3,216 \\
(46.2) \\
\end{array}$ & $\begin{array}{c}806 / 1474 \\
(54.7) \\
668 / 1,474 \\
(45.3) \\
\end{array}$ & $\begin{array}{c}533 / 978 \\
(54.5) \\
445 / 978 \\
(45.5) \\
\end{array}$ & $\begin{array}{c}232 / 444 \\
(52.3) \\
212 / 444 \\
(47.7)\end{array}$ \\
\hline $\begin{array}{l}\text { Length of hospital stay, } d \text { median } \\
\text { (IQR) }\end{array}$ & $32(16-54)$ & $55(32-81)$ & $31(15-52)$ & $40(25-59)$ & $24(12-43)$ & $22(9-41)$ \\
\hline $\begin{array}{l}\text { Length of stay until first positive } \\
\text { blood culture, d, median (IQR) }\end{array}$ & $13(5-24)$ & $28(15-46)$ & $12(5-23)$ & $16(10-27)$ & $10(3-19)$ & $6(1-16)$ \\
\hline Province & & & & & & \\
\hline $\begin{array}{l}\text { Gauteng } \\
\text { Other }\end{array}$ & $\begin{array}{c}4,229 / 6,669 \\
(63.4) \\
2,440 / 6,669 \\
(36.6)\end{array}$ & $\begin{array}{c}680 / 794 \\
(85.6) \\
114 / 794 \\
(14.4) \\
\end{array}$ & $\begin{array}{c}3,549 / 5,875 \\
(60.4) \\
2,326 / 5,875 \\
(39.6) \\
\end{array}$ & $\begin{array}{c}1,651 / 2,600 \\
(63.5) \\
949 / 2,600 \\
(36.5) \\
\end{array}$ & $\begin{array}{c}736 / 1,353 \\
(54.4) \\
617 / 1,353 \\
(45.6) \\
\end{array}$ & $\begin{array}{c}323 / 598 \\
(54) \\
275 / 598 \\
(46) \\
\end{array}$ \\
\hline $\begin{array}{l}\text { Healthcare sector } \\
\text { Public }\end{array}$ & $\begin{array}{c}2,529 / 6,669 \\
(37.9)\end{array}$ & $\begin{array}{c}99 / 794 \\
(12.5)\end{array}$ & $\begin{array}{c}2,430 / 5,875 \\
(41.4)\end{array}$ & $599 / 2,600(23)$ & $\begin{array}{c}673 / 1,353 \\
(49.7)\end{array}$ & $\begin{array}{c}248 / 598 \\
(41.5)\end{array}$ \\
\hline Private & $\begin{array}{c}4,140 / 6,669 \\
(62.1)\end{array}$ & $\begin{array}{c}695 / 794 \\
(87.5) \\
\end{array}$ & $\begin{array}{c}3,445 / 5,875 \\
(58.6)\end{array}$ & $\begin{array}{c}2,001 / 2,600 \\
(77)\end{array}$ & $\begin{array}{c}680 / 1,353 \\
(50.3)\end{array}$ & $\begin{array}{c}350 / 598 \\
(58.5)\end{array}$ \\
\hline Hospital admission in past 12 mo & $\begin{array}{c}1,428 / 1,967 \\
(72.6)\end{array}$ & $\begin{array}{c}77 / 104 \\
(74)\end{array}$ & $\begin{array}{c}1,351 / 1,863 \\
(72.5)\end{array}$ & $\begin{array}{c}378 / 529 \\
(71.5)\end{array}$ & $\begin{array}{c}341 / 486 \\
(70.2)\end{array}$ & $\begin{array}{c}126 / 174 \\
(72.4)\end{array}$ \\
\hline Intensive care unit admission & $\begin{array}{c}1,579 / 2,167 \\
(72.9)\end{array}$ & $\begin{array}{c}110 / 125 \\
(88)\end{array}$ & $\begin{array}{c}1,469 / 2,042 \\
(71.9)\end{array}$ & $\begin{array}{c}502 / 606 \\
(82.8)\end{array}$ & $\begin{array}{c}377 / 539 \\
(69.9)\end{array}$ & $\begin{array}{c}133 / 190 \\
(70)\end{array}$ \\
\hline Mechanical ventilation & $\begin{array}{l}611 / 1,818 \\
(33.6)\end{array}$ & $\begin{array}{l}44 / 91 \\
(48.4)\end{array}$ & $\begin{array}{l}567 / 1,727 \\
(32.8)\end{array}$ & $\begin{array}{c}175 / 476 \\
(36.8)\end{array}$ & $\begin{array}{c}129 / 440 \\
(29.3)\end{array}$ & $\begin{array}{c}57 / 165 \\
(34.6)\end{array}$ \\
\hline Central venous catheter in situ & $\begin{array}{c}1,031 / 1,817 \\
(56.7)\end{array}$ & $\begin{array}{c}69 / 92 \\
(75)\end{array}$ & $\begin{array}{l}962 / 1,725 \\
(55.8)\end{array}$ & $\begin{array}{c}289 / 479 \\
(60.3)\end{array}$ & $\begin{array}{c}229 / 443 \\
(51.7)\end{array}$ & $\begin{array}{c}89 / 165 \\
(53.9)\end{array}$ \\
\hline $\begin{array}{l}\text { Systemic antimicrobial drug } \\
\text { therapy in } 14 \mathrm{~d} \text { before positive } \\
\text { culture }\end{array}$ & $\begin{array}{l}1,292 / 1,830 \\
(70.6)\end{array}$ & $\begin{array}{l}77 / 94 \\
(81.9)\end{array}$ & $\begin{array}{c}1,215 / 1,736 \\
(70)\end{array}$ & $\begin{array}{c}349 / 481 \\
(72.6)\end{array}$ & $\begin{array}{c}284 / 441 \\
(64.4)\end{array}$ & $\begin{array}{c}105 / 164 \\
(64.0)\end{array}$ \\
\hline $\begin{array}{l}\text { Crude in-hospital case-fatality } \\
\text { ratio }\end{array}$ & $\begin{array}{c}8,39 / 1,966 \\
(42.7)\end{array}$ & $\begin{array}{l}46 / 102 \\
(45.1)\end{array}$ & $\begin{array}{c}793 / 1,864 \\
(42.5)\end{array}$ & $\begin{array}{c}166 / 516 \\
(32.2)\end{array}$ & $\begin{array}{c}247 / 492 \\
(50.2)\end{array}$ & $\begin{array}{l}91 / 179 \\
(50.8)\end{array}$ \\
\hline
\end{tabular}

\section{Discussion}

In conducting this comprehensive national survey, we found that $C$. auris caused $>10 \%$ of all cases of candidemia in South Africa and was the third most common Candida species. The incidence of $C$. auris candidemia was highest in private-sector hospitals in Gauteng Province. The crude in-hospital case-fatality ratio did not differ between Candida species. Prior systemic antifungal drug therapy was associated with increased adjusted odds of $C$. auris fungemia compared with candidemia caused by other species, and this effect was stronger in public-sector hospitals.

C. auris has rapidly emerged as a major cause of candidemia in South Africa, surpassing the number of cases caused by C. glabrata, C. tropicalis, and C. krusei over the past 7 years. A clear shift has occurred in the epidemiology observed from a previous national survey during 2009-
2010 and a recent dramatic increase in the number of cases of $C$. auris invasive infection and colonization nationwide $(1,12)$. We speculate that delayed clinician and laboratory awareness might have led to undetected transmission of the pathogen early in the epidemic (16).

The incidence of $C$. auris candidemia was highest in hospitals in Gauteng Province and is partly attributable to ongoing and recurrent clusters in these hospitals during the surveillance period. We speculate that the epidemic in South Africa might be centered in this area because of a combination of complex and interdependent healthcare system and behavioral factors, including a highly concentrated and mobile patient population; a large number of referrals and admission of patients with clinically complex cases to hospitals in the region; indiscriminate use of antimicrobial agents, including azoles and 


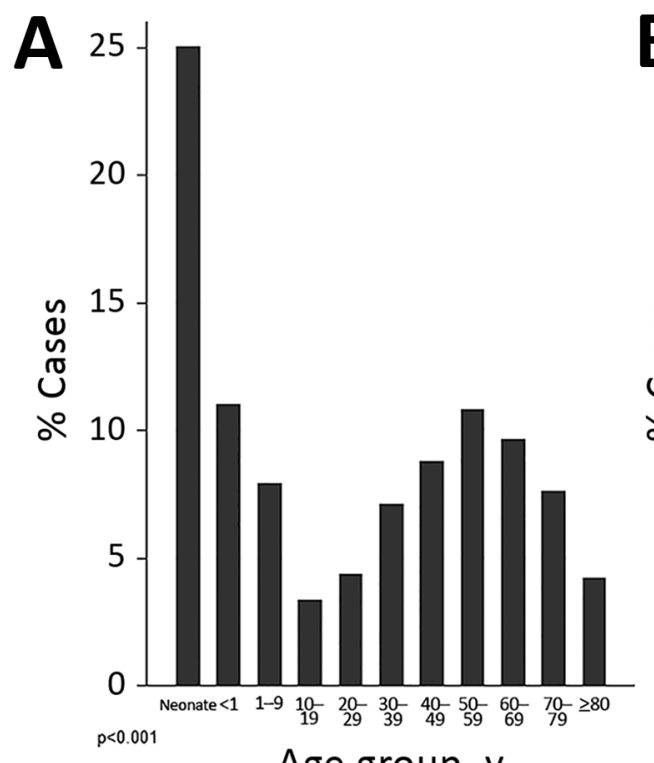

Age group, $y$
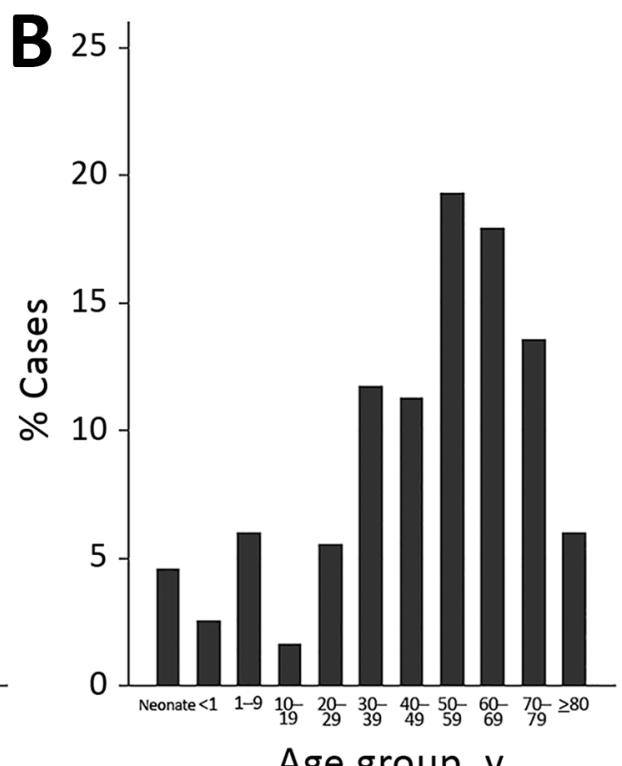

Figure 2. Age distribution of case-patients with candidemia caused by Candida auris compared with other Candida species, South Africa, 20162017. A) C. auris patient median age was 54 years (interquartile range 34-67 years); B) other Candida species patient median age was 27 years (interquartile range $0-57$ years). echinocandins; and suboptimal infection prevention and control practices. In addition, international travel to and from Gauteng Province might also play a role, as suggested by recent case reports and outbreaks in other continents caused by the South Africa clade of C. auris $(5,17-20)$. In the United States, $90 \%$ of clinical cases of $C$. auris occurred in the New York metropolitan area, and most patients had lengthy hospitalizations in facilities that had capacity for highly skilled nursing and mechanical ventilation (21), suggesting that a large susceptible population of severely ill patients within a facility might provide a starting point for an outbreak that is then amplified by transmission. Individual hospital outbreaks seemed to overlap in Gauteng Province, suggesting that interfacility and intersectoral transmission of infections might have occurred; however, we have not yet established epidemiologic links among cases from different facilities. Whole-genome sequencing to establish molecular links is under way to more clearly characterize the epidemiology of $C$. auris candidemia in South Africa.

Prior systemic antifungal drug use was associated with $C$. auris candidemia, particularly in public-sector

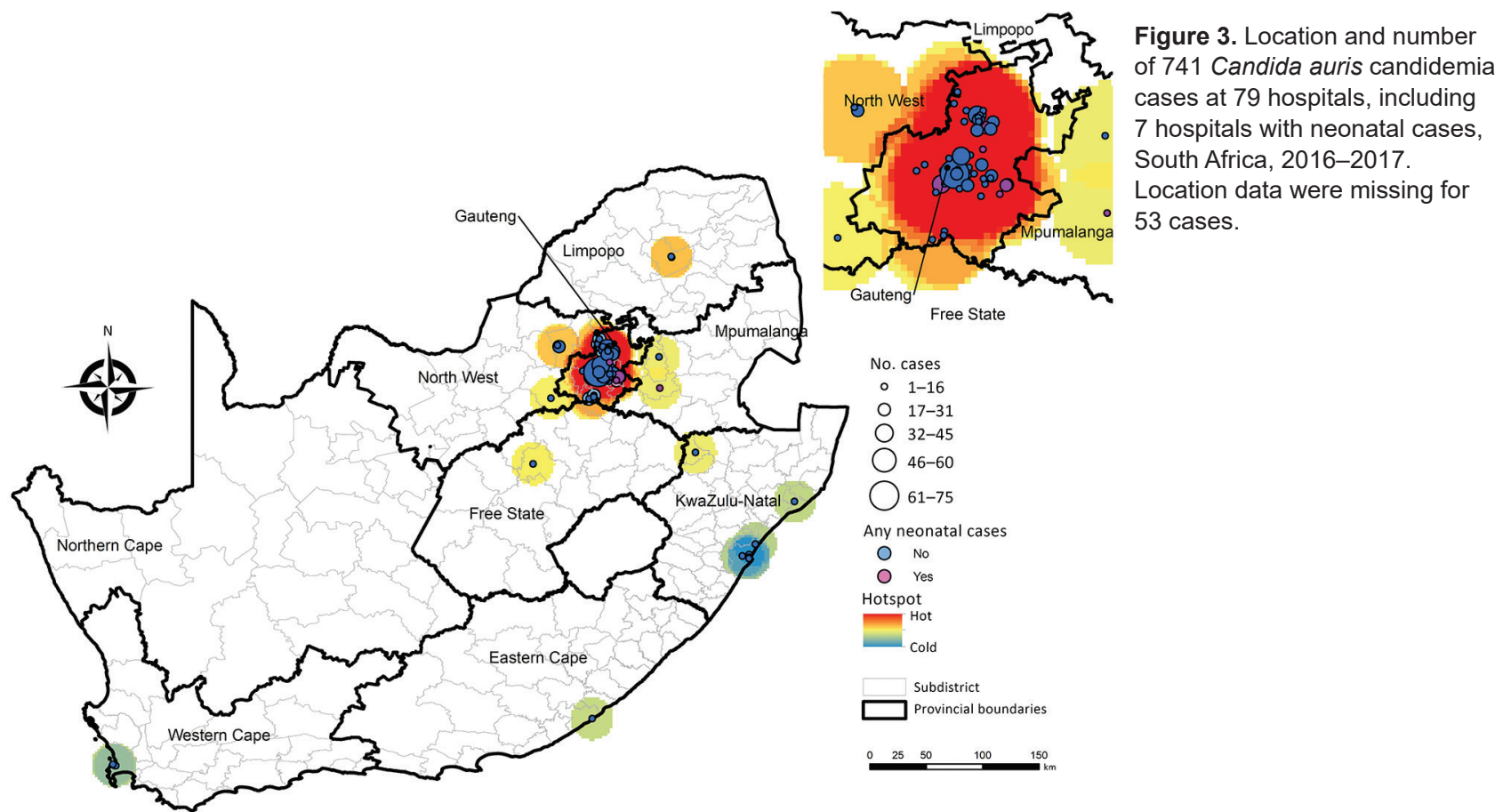




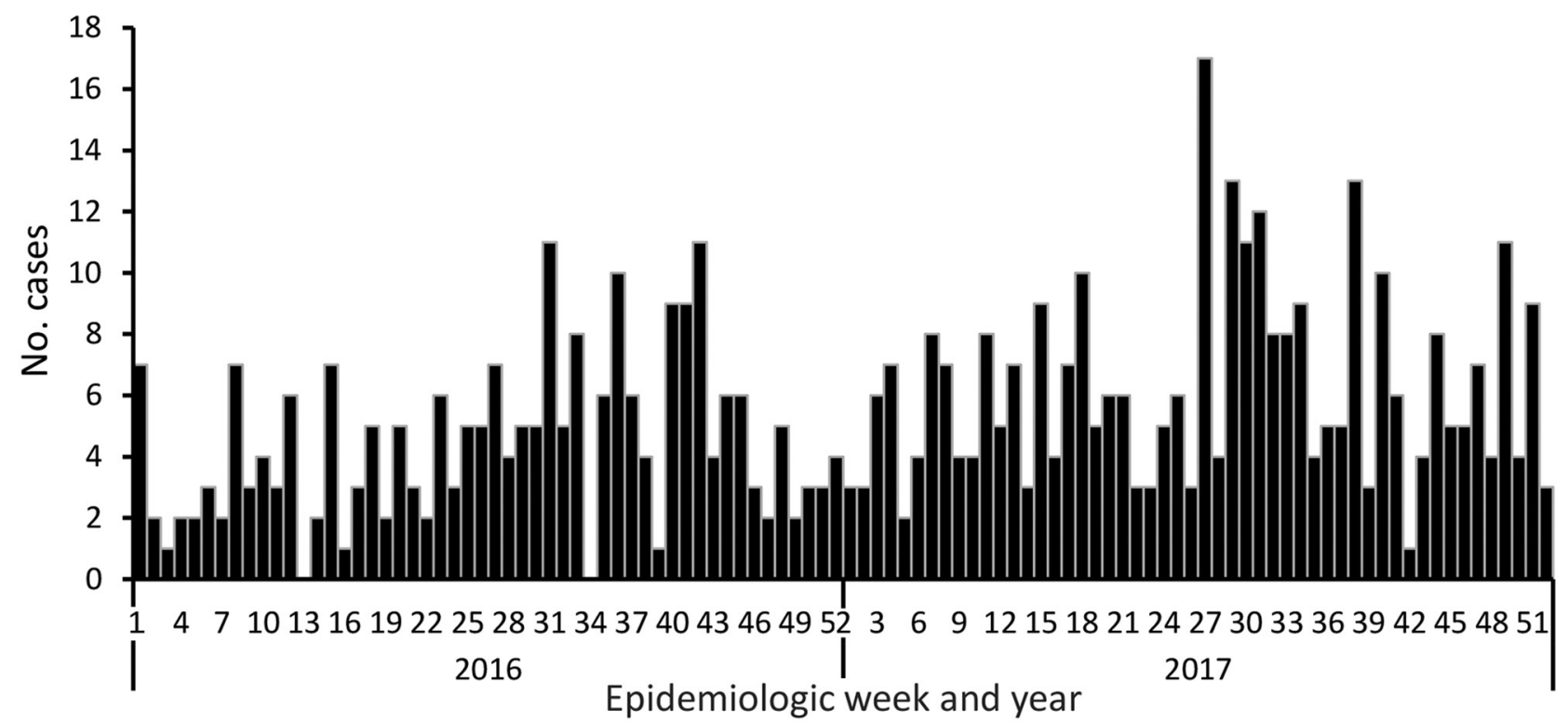

Figure 4. Cases of Candida auris candidemia $(N=557)$, by epidemiologic week, Gauteng Province, South Africa, 2016-2017. Date of blood culture collection was missing for 123 cases.

hospitals. This finding is consistent with data from similar studies and is probably related to selective pressure by azoles (6). Almost all tested C. auris isolates from South Africa are resistant to fluconazole (2) (T.G. Maphanga, NICD, pers. comm., 2018 Jul 27). Fluconazole is commonly used as a first-line treatment option, especially in public-sector hospitals, where access to echinocandin antifungal drugs is currently limited. The forthcoming 2019 guidelines for treatment of $C$. auris in South Africa recommend echinocandins as a first-line treatment for candidemia and amphotericin $\mathrm{B}$ deoxycholate if echinocandins are unavailable (22). In contrast to other Candida species, such as C. parapsilosis, for which a substantial proportion of infections occur among the neonatal population, C. auris occurs among older adults (12). In South Africa, an outbreak among 6 neonates in a neonatal unit has been documented (23), and several other small outbreaks have occurred (N.P. Govender, unpub. data). To date, no neonatal cases have been reported from the United States or Europe, although India, Colombia, and Venezuela have reported cases $(4-6,19,24,25)$. Whether this phenomenon

Table 3. Demographic and clinical characteristics of patients with Candida auris candidemia, by healthcare sector, South Africa, 2016-2017*

\begin{tabular}{|c|c|c|c|}
\hline Characteristic & $\begin{array}{c}\text { Public-sector } \\
\text { hospitals, } \mathrm{n}=99\end{array}$ & $\begin{array}{c}\text { Private-sector } \\
\text { hospitals, } n=695\end{array}$ & $\mathrm{p}$ value \\
\hline Age, y, median (IQR) & $27(2-42)$ & $58(44-70)$ & $<0.001$ \\
\hline Sex & & & 0.64 \\
\hline Men and boys & $63 / 99(64)$ & $221 / 364(61)$ & NA \\
\hline Women and girls & $36 / 99(36)$ & $143 / 364(39)$ & NA \\
\hline Length of hospital stay, d, median (IQR) & $49(30-72)$ & $68(40-140)$ & 0.03 \\
\hline Length of stay to first positive blood culture, $d$, median (IQR) & $26(13-42)$ & $35(16-58)$ & 0.21 \\
\hline Hospital admission in past $12 \mathrm{mo}$ & $37 / 62(60)$ & 40/42 (95) & $<0.001$ \\
\hline Intensive care unit admission & $54 / 68(79.4)$ & $56 / 57(98.3)$ & 0.001 \\
\hline Mechanical ventilation & $21 / 52(40)$ & $23 / 39(59)$ & 0.09 \\
\hline Central venous catheter in situ & $40 / 54(74)$ & 29/38 (76) & 1.0 \\
\hline Total parenteral nutrition & $22 / 52(42)$ & $15 / 38(39)$ & 0.83 \\
\hline Systemic antimicrobial drug therapy $\leq 14 \mathrm{~d}$ before positive culture & $36 / 52(69)$ & $41 / 42(98)$ & $<0.001$ \\
\hline Systemic antifungal drug therapy $\leq 14 \mathrm{~d}$ before positive culture $\neq$ & $14 / 53(26)$ & $16 / 42(38)$ & 0.27 \\
\hline Azole & $12 / 14(85.7)$ & $4 / 16(25)$ & 0.001 \\
\hline Polyene/amphotericin B & $4 / 14(28.6)$ & $3 / 16(18.8)$ & 0.68 \\
\hline Echinocandin & $0 / 14(0)$ & $13 / 16(81.3)$ & $<0.001$ \\
\hline Crude in-hospital mortality ratio & $22 / 59(37)$ & $24 / 43(56)$ & 0.07 \\
\hline
\end{tabular}

*Values are no. (\%) except as indicated. Age data were available for 435 patients, and data on sex were available for 428 patients. For the rest of the variables, data were available for only a small proportion of patients from enhanced surveillance sites (total, $N=110 ;$ public sector, $n=67 ;$ private sector, $n=43)$. IQR, interquartile range; NA, not applicable.

†Proportions were compared by using a $\chi^{2}$ or Fisher exact test; medians were compared by using the Wilcoxon rank-sum test.

$\ddagger$ Patients could have received $>1$ class of antifungal drug therapy. 
is attributable to inherent factors of the pathogen, environmental factors in neonatal units, or chance is still unclear. Nevertheless, we should be proactive to not let $C$. auris establish a foothold in neonatal units in developing countries as C. parapsilosis has done $(12,26)$.

In the unique healthcare environment of South Africa, patients admitted to private-sector facilities were more likely to have $C$. auris candidemia. We hypothesize that this might be attributable to early undetected outbreaks in this sector, inherent differences in the patient populations admitted, or structural differences in the 2 healthcare sectors; more patients with $C$. auris candidemia at privatesector facilities were mechanically ventilated, had prior hospitalization, and had prior systemic antimicrobial drug therapy. Antimicrobial drug prescription behavior and differences in antimicrobial drug stewardship practices, including easier access to a broader range of antifungal drugs, might also play a role. Last, ongoing outbreaks at a few facilities might drive the higher case numbers in the private healthcare sector. The presence of a central venous catheter is a well-established risk factor for bloodstream infections (27). It is not surprising that central venous catheters were associated with $C$. auris candidemia because the pathogen has been shown to form biofilms and adhere to polymeric surfaces $(8,10)$.

To address the continued transmission of $C$. auris in health facilities in South Africa, C. auris has been identified as a priority pathogen for surveillance to monitor emergence of antifungal drug resistance from all infection sites. We have also adapted published laboratory methods for rapid identification of $C$. auris colonization in the context of outbreak investigations (28). Local studies are also being planned to investigate the efficacy of novel antifungal agents (29).

This study had several limitations. We analyzed data for laboratory-confirmed candidemia only and did not include patients with other invasive Candida infections, culture-negative sepsis, or colonization, which might underestimate the extent of the problem in South Africa. However, $18 \%-22 \%$ of reported cases of C. auris infection in Europe and South Africa are bloodstream infections, and $58 \%$ of clinical isolates in the United States are from blood $(1,19,30)$. In addition, $77 \%$ of cases of $C$. auris infection reported in the international literature are cases of candidemia; therefore, our study provides a plausible representation of the epidemiology of $C$. auris, albeit just the proverbial tip of the iceberg (3). The determination of incidence risk was based on data from a limited number of hospitals with admissions data available, mostly from the private sector. Therefore, we might have underestimated the incidence risk in the public sector. The reference laboratory confirmed the species identification of bloodstream isolates from only $45 \%$ of all detected cases of candidemia.
Most cases without a reference laboratory species identification $(70 \%)$ were from the private sector and had been detected retrospectively through audits. However, we believe that these national surveillance data still provide an accurate representation of the actual distribution of $C$. auris candidemia cases across sectors because most private laboratories used MALDI-TOF mass spectrometry methods to confirm Candida species identification. For cases at enhanced surveillance sites, we were limited to the availability of secondary data collected through an established surveillance program; we were unable to assess the duration of exposure to certain factors, such as parenteral nutrition and type of prior antimicrobial drug exposure. In addition, the linking of audit cases to reported cases was limited by demographic data available; therefore, we might have included duplicate cases in our analysis. Misclassification error might have occurred, given that a proportion of isolates did not have a species-level identification.

C. auris was the third most common cause of candidemia in South Africa and caused $14 \%$ of all cases during 2016-2017. Ongoing and recurrent micro-outbreaks might have driven the larger epidemic centered in Gauteng Province. Individual patient and healthcare risk factors should be considered when managing patients with suspected candidemia. The use of molecular epidemiology is needed to further characterize outbreaks in South Africa and better understand transmission dynamics of this emerging pathogen.

\section{Acknowledgments}

We are grateful to Jimmy Khosa for designing the maps included in this paper. We acknowledge Briette du Toit, Angeliki Messina, Hleziphi Mahlangu, and Joy Cleghorn for providing denominator data for the major private-sector hospital groups. We also acknowledge the following contributors to the GERMS-SA candidemia surveillance programme for participating in surveillance and sending Candida isolates to the National Institute for Communicable Diseases: John Black, Shareef Abrahams, Vanessa Pearce, Anwar Hoosen, Vicky Kleinhans, Masego Moncho, Alan Karstaedt, Caroline Maluleka, Charl Verwey, Charles Feldman, David Moore, Gary Reubenson, Khine Swe Swe Han, Jeannette Wadula, Jeremy Nel, Kathy Lindeque, Maphoshane Nchabeleng, Nazlee Samodien, Nicolette du Plessis, Nontombi Mbelle, Nontuthuko Maningi, Norma Bosman, Ranmini Kularatne, Sharona Seetharam, Teena Thomas, Theunis Avenant, Trusha Nana, Vindana Chibabhai, Adhil Maharj, Asmeeta Burra, Fathima Naby, Halima Dawood, Jade Mogamberry, Koleka Mlisana, Lisha Sookan, Praksha Ramjathan, Prasha Mahabeer, Romola Naidoo, Sumayya Haffejee, Yacoob Coovadia Khine Swe Swe Han, Nomonde Dlamini, Surendra Sirkar, Ken Hamese, Ngoaka Sibiya, Ruth Lekalakala, Greta Hoyland, Jacob Lebudi, Pieter Jooste, Ebrahim Variava, Erna du Plessis, Andrew Whitelaw, 
Kessendri Reddy, Mark Nicol, Preneshni Naicker, Colleen Bamford, Adrian Brink, Ebrahim Hoosien, Elizabeth Prentice, Inge Zietsman, Maria Botha, Peter Smith, Terry Marshall, Xoliswa Poswa, Chetna Govind, Juanita Smit, Keshree Pillay, Sharona Seetharam, Suzy Budavari, Victoria Howell, Carel Haumann, Catherine Samuel, Marthinus Senekal, Andries Dreyer, Khatija Ahmed, Louis Marcus, Warren Lowman, Angeliki Messina, Dena van den Bergh, and Karin Swart. We also acknowledge our NICD GERMS-SA colleagues: Ananta Nanoo, Andries Dreyer, Anne von Gottberg, Anthony Smith, Arvinda Sooka, Cecilia Miller, Charlotte Sriruttan, Cheryl Cohen, Chikwe Ihekweazu, Claire von Mollendorf, Desiree du Plessis, Erika van Schalkwyk, Farzana Ismail, Frans Radebe, Genevie Ntshoe, Gillian Hunt, Hlengani Mathema, Husna Ismail, Jacqueline Weyer, Jackie Kleynhans, Jenny Rossouw, John Frean, Joy Ebonwu, Judith Mwansa-Kambafwile, Karen Keddy, Kerrigan McCarthy, Liliwe Shuping, Linda de Gouveia, Linda Erasmus, Lucille Blumberg, Marshagne Smith, Martha Makgoba, Motshabi Modise, Nazir Ismail, Nelesh Govender, Neo Legare, Nicola Page, Ntsieni Ramalwa, Nuraan Paulse, Phumeza Vazi, Olga Perovic, Penny Crowther-Gibson, Portia Mutevedzi, Riyadh Manesen, Ranmini Kularatne, Ruth Mpembe, Sarona Lengana, Shabir Madhi, Shaheed Vally Omar, Sibongile Walaza, Sonwabo Lindani, Sunnieboy Njikho, Susan Meiring, Thejane Motladiile, Tiisetso Lebaka, Vanessa Quan, and Verushka Chetty. Finally, we acknowledge persons from the Centre for Healthcare-Associated Infections, Antimicrobial Resistance, and Mycoses at NICD: Serisha Naicker, Tsidiso Maphanga, Mabatho Mhlanga, Thokozile Gloria Zulu, Ernest Tsotetsi, Phelly Matlapeng, Siphiwe Kutta, Lerato Qoza, Sydney Mogokotleng, Mbali Dube, and Amanda Shilubane.

This work was supported in its entirety by NICD, a division of NHLS, in Johannesburg, South Africa.

\section{About the Author}

Dr. van Schalkwyk is a medical epidemiologist at the National Institute for Communicable Diseases, South Africa. Her research interests include mycoses, healthcare-associated infections, and antimicrobial resistance.

\section{References}

1. Govender NP, Magobo RE, Mpembe R, Mhlanga M, Matlapeng P, Corcoran C, et al. Candida auris in South Africa, 2012-2016. Emerg Infect Dis. 2018;24:2036-40. http://dx.doi.org/ 10.3201/eid2411.180368

2. Lockhart SR, Etienne KA, Vallabhaneni S, Farooqi J, Chowdhary A, Govender NP, et al. Simultaneous emergence of multidrug-resistant Candida auris on 3 continents confirmed by whole-genome sequencing and epidemiological analyses. Clin Infect Dis. 2017;64:134-40. http://dx.doi.org/10.1093/cid/ ciw691

3. Jeffery-Smith A, Taori SK, Schelenz S, Jeffery K, Johnson EM, Borman A, et al.; Candida auris Incident Management Team. Candida auris Incident Management Team. Candida auris: a review of the literature. Clin Microbiol Rev. 2017;31:e0029-17. http://dx.doi.org/10.1128/CMR.00029-17

4. Schelenz S, Hagen F, Rhodes JL, Abdolrasouli A, Chowdhary A, Hall A, et al. First hospital outbreak of the globally emerging Candida auris in a European hospital. Antimicrob Resist Infect Control. 2016;5:35. http://dx.doi.org/10.1186/ s13756-016-0132-5

5. Ruiz-Gaitán A, Moret AM, Tasias-Pitarch M, Aleixandre-López AI, Martínez-Morel H, Calabuig E, et al. An outbreak due to Candida auris with prolonged colonisation and candidaemia in a tertiary care European hospital. Mycoses. 2018;61:498-505. http://dx.doi.org/10.1111/myc.12781

6. Rudramurthy SM, Chakrabarti A, Paul RA, Sood P, Kaur H, Capoor MR, et al. Candida auris candidaemia in Indian ICUs: analysis of risk factors. J Antimicrob Chemother. 2017;72:1794801. http://dx.doi.org/10.1093/jac/dkx034

7. Ku TSN, Walraven CJ, Lee SA. Candida auris: disinfectants and implications for infection control. Front Microbiol. 2018;9:726. http://dx.doi.org/10.3389/fmicb.2018.00726

8. Welsh RM, Bentz ML, Shams A, Houston H, Lyons A, Rose LJ, et al. Survival, persistence, and isolation of the emerging multidrug-resistant pathogenic yeast Candida auris on a plastic health care surface. J Clin Microbiol. 2017;55:2996-3005. http://dx.doi.org/10.1128/JCM.00921-17

9. Piedrahita CT, Cadnum JL, Jencson AL, Shaikh AA, Ghannoum MA, Donskey CJ. Environmental surfaces in healthcare facilities are a potential source for transmission of Candida auris and other Candida species. Infect Control Hosp Epidemiol. 2017;38:1107-9. http://dx.doi.org/10.1017/ice.2017.127

10. Sherry L, Ramage G, Kean R, Borman A, Johnson EM, Richardson MD, et al. Biofilm-forming capability of highly virulent, multidrug-resistant Candida auris. Emerg Infect Dis. 2017;23:328-31. http://dx.doi.org/10.3201/eid2302.161320

11. Magill SS, O'Leary E, Janelle SJ, Thompson DL, Dumyati G, Nadle J, et al.; Emerging Infections Program Hospital Prevalence Survey Team. Changes in prevalence of health care-associated infections in U.S. hospitals. N Engl J Med. 2018;379:1732-44. http://dx.doi.org/10.1056/NEJMoa1801550

12. Govender NP, Patel J, Magobo RE, Naicker S, Wadula J, Whitelaw A, et al.; TRAC-South Africa group. Emergence of azole-resistant Candida parapsilosis causing bloodstream infection: results from laboratory-based sentinel surveillance in South Africa. J Antimicrob Chemother. 2016;71:1994-2004. http://dx.doi.org/10.1093/jac/dkw091

13. Cornely OA, Bassetti M, Calandra T, Garbino J, Kullberg BJ, Lortholary O, et al.; ESCMID Fungal Infection Study Group. ESCMID guideline for the diagnosis and management of Candida diseases 2012: non-neutropenic adult patients. Clin Microbiol Infect. 2012;18(Suppl 7):19-37. http://dx.doi.org/10.1111/ 1469-0691.12039

14. Kreusch A, Karstaedt AS. Candidemia among adults in Soweto, South Africa, 1990-2007. Int J Infect Dis. 2013;17:e621-3. http://dx.doi.org/10.1016/j.ijid.2013.02.010

15. Econex. The South African private healthcare sector: role and contribution to the economy [cited 2019 Jan 9]. https://econex.co.za/wp-content/uploads/2016/09/ Econex_private_health_sector_study_12122013-1.pdf

16. Magobo RE, Corcoran C, Seetharam S, Govender NP. Candida auris-associated candidemia, South Africa. Emerg Infect Dis. 2014;20:1250-1. http://dx.doi.org/10.3201/eid2007.131765

17. Eyre DW, Sheppard AE, Madder H, Moir I, Moroney R, Quan TP, et al. A Candida auris outbreak and its control in an intensive care setting. N Engl J Med. 2018;379:1322-31. http://dx.doi.org/10.1056/NEJMoa1714373

18. Belkin A, Gazit Z, Keller N, Ben-Ami R, Wieder-Finesod A, Novikov A, et al. Candida auris infection leading to nosocomial 
transmission, Israel, 2017. Emerg Infect Dis. 2018;24:801-4. http://dx.doi.org/10.3201/eid2404.171715

19. Chow NA, Gade L, Tsay SV, Forsberg K, Greenko JA, Southwick KL, et al.; US Candida auris Investigation Team. Multiple introductions and subsequent transmission of multidrugresistant Candida auris in the USA: a molecular epidemiological survey. Lancet Infect Dis. 2018;18:1377-84. http://dx.doi.org/ 10.1016/S1473-3099(18)30597-8

20. Heath CH, Dyer JR, Pang S, Coombs GW, Gardam DJ. Candida auris sternal osteomyelitis in a man from Kenya visiting Australia, 2015. Emerg Infect Dis. 2019;25:192-4. http://dx.doi.org/10.3201/ eid2501.181321

21. Tsay S, Welsh RM, Adams EH, Chow NA, Gade L, Berkow EL, et al. Ongoing transmission of Candida auris in health care facilities-United States, June 2016-May 2017. MMWR Morb Mortal Wkly Rep. 2017;66:514-5. http://dx.doi.org/10.15585/ mmwr.mm6619a7

22. Govender NP, Avenant T, Brink A, Chibabhai V, Cleghorn J, du Toit B, et al. FIDSSA guideline: recommendations for detection, management and prevention of healthcare-associated Candida auris colonisation and disease in South Africa S. Afr J Infect Dis. 2019. In press.

23. Moema I, Ismail H, van Schalkwyk E, Shuping L, Govender NP. Outbreak of culture-confirmed Candida auris bloodstream infection in the neonatal unit of a public-sector hospital, South Africa, July through September 2017. In: Abstracts of the 67th Annual Epidemic Intelligence Service Conference; Atlanta; 2018 April 16-19 [cited 2018 Sep 28]. https://www.cdc.gov/eis/ downloads/eis-conference-2018-508.pdf

24. Escandón P, Cáceres DH, Espinosa-Bode A, Rivera S, Armstrong P, Vallabhaneni S, et al. Surveillance for Candida auris - Colombia, September 2016-May 2017. MMWR Morb Mortal Wkly Rep. 2018;67:459-60. http://dx.doi.org/10.15585/ mmwr.mm6715a6

25. Calvo B, Melo AS, Perozo-Mena A, Hernandez M, Francisco EC, Hagen F, et al. First report of Candida auris in America: Clinical and microbiological aspects of 18 episodes of candidemia. J Infect. 2016;73:369-74. http://dx.doi.org/10.1016/j. jinf.2016.07.008

26. Pammi M, Holland L, Butler G, Gacser A, Bliss JM. Candida parapsilosis is a significant neonatal pathogen: a systematic review and meta-analysis. Pediatr Infect Dis J. 2013;32:e206-16. http://dx.doi.org/10.1097/INF.0b013e3182863a1c

27. Crnich CJ, Maki DG. The role of intravascular devices in sepsis. Curr Infect Dis Rep. 2001;3:496-506. http://dx.doi.org/10.1007/ s11908-001-0086-4

28. Sexton DJ, Kordalewska M, Bentz ML, Welsh RM, Perlin DS, Litvintseva AP. Direct detection of emergent fungal pathogen Candida auris in clinical skin swabs by SYBR green-based quantitative PCR assay. J Clin Microbiol. 2018;56:e01337-18. http://dx.doi.org/10.1128/JCM.01337-18

29. Berkow EL, Lockhart SR. Activity of novel antifungal compound APX001A against a large collection of Candida auris. J Antimicrob Chemother. 2018;73:3060-2. http://dx.doi.org/ 10.1093/jac/dky302

30. Kohlenberg A, Struelens MJ, Monnet DL, Plachouras D; The Candida Auris Survey Collaborative Group. Candida auris: epidemiological situation, laboratory capacity and preparedness in European Union and European Economic Area countries, 2013 to 2017. Euro Surveill. 2018;23:23. http://dx.doi.org/10.2807/ 1560-7917.ES.2018.23.13.18-00136

Address for correspondence: Nelesh P. Govender, National Institute for Communicable Diseases, 1 Modderfontein Rd, Sandringham, Johannesburg, 2131, South Africa; email: neleshg@nicd.ac.za

\section{EID podcast} Developing Biological Reference Materials

\section{to Prepare for Epidemics}

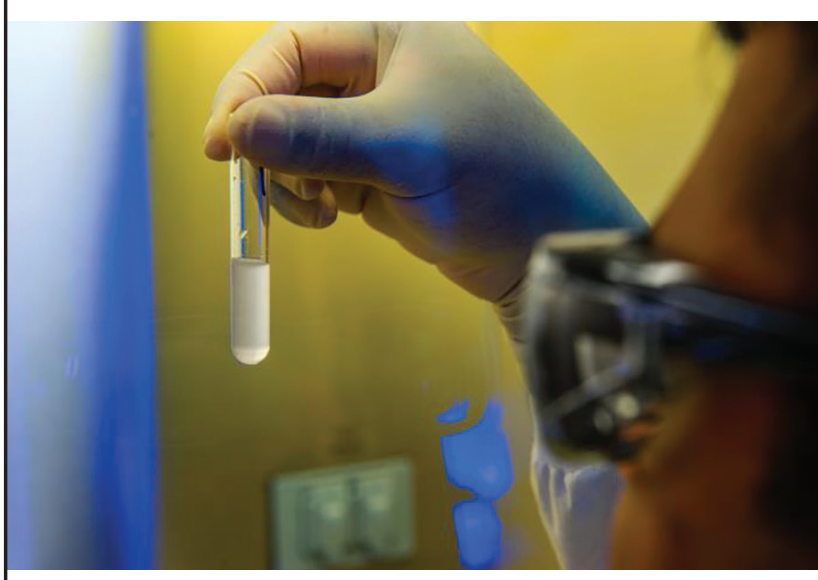

Having standard biological reference materials, such as antigens and antibodies, is crucial for developing comparable research across international institutions. However, the process of developing a standard can be long and difficult.

In this EID podcast, Dr. Tommy Rampling, a clinician and academic fellow at the Hospital for Tropical Diseases and University College in London, explains the intricacies behind the development and distribution of biological reference materials.

\section{Visit our website to listen: https://go.usa.gov/xyfJX}

\section{EMERGING \\ INFECTIOUS DISEASES}

